OPEN ACCESS

Edited by:

Aida Martin,

Quantitative BioSciences, Inc.,

United States

Reviewed by:

Itthipon Jeerapan,

Prince of Songkla University, Thailand

Gregoire Thouvenin,

University of California, San Diego,

United States

Fernando Soto,

Stanford University, United States

*Correspondence:

Weinan Xu

weinanxu@uakron.edu

Specialty section:

This article was submitted to

Biosensors,

a section of the journal

Frontiers in Sensors

Received: 22 July 2020

Accepted: 07 September 2020

Published: 02 October 2020

Citation:

Liu S and Xu W (2020) Engineered Living Materials-Based Sensing and Actuation. Front. Sens. 1:586300. doi: 10.3389/fsens. 2020.586300

\section{Engineered Living Materials-Based Sensing and Actuation}

\author{
Shan Liu and Weinan $\mathrm{Xu}$ * \\ School of Polymer Science and Polymer Engineering, University of Akron, Akron, $\mathrm{OH}$, United States
}

The integration of functional synthetic materials and living biological entities has emerged as a new and powerful approach to create adaptive and functional structures with unprecedented performance and functionalities. Such hybrid structures are also called engineered living materials (ELMs). ELMs have the potential to realize many highly-desired properties, which are usually only found in biological systems, such as the abilities to self-power, self-heal, response to biosignals, and self-sustainable. Motivated by that, in recent years, researchers have started to explore the use of ELMs in many areas, among them, sensing and actuation is the area that has seen the most progress. In this short review, we briefly reviewed the important recent development in ELMs-based sensors and actuators, with a focus on their materials and structural design, new fabrication technologies, and bio-related applications. Current challenges and future directions in this field are also identified to help with future development in this emerging interdisciplinary field.

Keywords: engineered living materials, sensing, actuation, stimuli-responsive, 3D printing

\section{INTRODUCTION}

One of the most sought-after objectives in materials science is to create a material system that possesses many unique advantages of the biological systems, including self-sustainable, selfrepair, self-replicating, and biosignal-responsive (Gilbert and Ellis, 2019). To realize this long-term objective, in recent years, the concept of engineered living materials (ELMs) has drawn significant attention (Nguyen et al., 2018; Guo et al., 2020). ELMs are an emerging class of materials that combine living biological entities with functional soft materials (Chen et al., 2015; Heyde et al., 2017; Ruan et al., 2020). The incorporation of biological cells or tissues provides the materials with biosensing, self-regenerative, and molecular computing capabilities (Huang et al., 2019; Pu et al., 2020). At the same time, the matrix or encapsulating materials provide the engineered biological entities with protection, mechanical enhancement, and communication pathways (CaroAstorga et al., 2020; Heveran et al., 2020). The synergic combination of living biological entities and functional soft materials enables ELMs with unprecedented properties and functionalities in many applications, especially in sensing and actuation.

The utilization of soft and adaptive materials in the fabrication of soft sensors and actuators has attracted significant attention in recent years (Polygerinos et al., 2017; Kim et al., 2018; Majidi, 2018). Those soft functional materials range from compliant conductors, semiconductors, to dielectrics (Chen and Pei, 2017; Miriyev et al., 2017; Arab Hassani et al., 2020); usually, a combination of electrically/optically active nanomaterials with adaptive soft matrices, such as stimuli-responsive or shape-memory polymers, is essential to achieve the desired properties (Bahramzadeh and Shahinpoor, 2014; Truby et al., 2018). Those soft sensors and actuators are 
able to sense temperature, $\mathrm{pH}$, ions, pressure, and touch (Bandodkar et al., 2016; Cao et al., 2017), or perform programmed locomotion, shape changes, or pick-and-place operations (Laschi et al., 2016; Cianchetti et al., 2018; Rich et al., 2018), which lays the foundation for electronic skins and soft robotics.

Despite the significant progress in soft sensors and actuators based on synthetic functional materials, their seamless integration with biological systems is still challenging due to the intrinsic differences in structures and properties between synthetic materials and biological cells/tissues (Chen et al., 2017; $\mathrm{Xu}$ et al., 2019). To bring new bio-relevant functionalities and generate self-powered or even self-regenerative next-generation soft sensors and actuators, one of the most promising approaches is by integrating those smart synthetic materials with living biological cells.

On the other hand, the use of microorganisms and biological cells for sensing applications have been explored for decades, the rapid advances in synthetic biology enables disease diagnosis and treatment, environmental monitoring, and toxicological screening, based on the information-processing abilities of living cells (Su et al., 2011; Bird et al., 2019; Inda and Lu, 2020). Those conventional biological cells-based biosensors are not within the scope of this paper, as they have already been summarized in recent review papers (Chang et al., 2017; Roggo and van der Meer, 2017). There are several excellent reviews on ELMs during the past several years, which are more comprehensive and have different emphases, such as engineered bacterial systems (Nguyen et al., 2018), biohybrid actuators (Ricotti et al., 2017), and soft robotics (Appiah et al., 2019). The focus of this mini review is to highlight the most recent advances in the integration of microorganisms and cells with functional soft materials for sensing and actuation, with emphasis on the cell-material integration and fabrication technologies.

In this mini review, we present a brief overview of the emerging area of ELMs-based sensing and actuation, with a focus on the hybrid materials design and composition, interfacial engineering, as well as new fabrication technologies. We first discuss ELMs-based structure for biosensing, in which the biological cells are the signal generating, transducing or transmitting component, and supported by the soft functional matrices. Then we discuss ELMs-based structure for actuation; the living biological cells/tissues can either directly generate mechanical force through contraction or generate chemical signals to activate other functional components. Finally, we summarize the progress and challenges in this emerging field, point out the topics and areas where future research endeavors are most likely to make breakthroughs.

\section{ELMS-BASED STRUCTURES FOR BIOSENSING}

The integration of biological cells/tissues with soft functional materials can be achieved in various ways. One of the simplest strategies to fabricate ELMs-based biosensor is by encapsulating biological cells inside hydrogel particles (Velasco et al., 2012; Le Goff et al., 2015; Culver et al., 2018). The encapsulation of biological cells in hydrogel generally improves the long-term viability/activity of the biological sensing agent, also the portability and applicability of the bioassay system. For instance, copolymer hydrogel microbeads were used to encapsulate genetically engineered $E$. coli cells (Kim et al., 2019), which can be used for the detection of nitro compounds such as TNT through the fluorescent signal amplification of the E. coli. The strong affinity between the E. coli and the copolymer enables significantly enhanced fluorescent signals and sensitivity. In another report (Zhang et al., 2019a), hydrogel encapsulated E. coli cells were used as the sensor for heavy metal ions based on the enzymatic activity of the cells. Genetically engineered bacteria were also embedded in a vesicle-based cell mimic (Trantidou et al., 2018), and used as biosensing modules for real-time monitoring of lactate in the environment.

The fabrication of well-defined ELM microparticles on a large scale requires new fabrication methods; at the same time, the fabrication techniques need to be compatible with biological cells. For instance, microfluidic fabrication was used to generated hydrogel microparticles with genetically engineered lactam-responsive $E$. coli encapsulated inside (Ma et al., 2019), and the microparticles can be used for detection of lactam species in a dose-dependent manner. Electrostatic extrusion was used for the fabrication of bacteria loaded alginate-methacrylate hydrogel beads ( $\mathrm{Li}$ et al., 2017), the encapsulated reporter bacteria responded with a strong fluorescence signal when exposed to the autoinducer molecules.

Simple embedding of biological cells inside a soft matrix has its limitations in structure and performance for more advanced biosensing, and it also suffers from issues such as cell leakage. In order to fabricate more advanced ELMs-based biosensors with higher sensitivity and stability, easier and more accurate data collection and analysis, it is necessary to integrate the biological cells, matrix materials, as well as other functional components in a more controlled way, for instance, using micropatterning or 3D fabrication techniques to spatially control the location of biological cells. To solve the problem of cell leakage from the hydrogel matrix, and fabricate flexible and stretchable devices (Liu et al., 2017), hydrogel-elastomer hybrids were developed that encapsulate multiple types of genetically engineered bacterial cells (Figures 1A,B). This design prevents leakage of cells from the biosensing devices even under large deformations. Stretchable living sensors that can respond to multiple biochemicals in several form factors such as skin patches (Figure 1C) were also demonstrated, cognate inducers were detected by the genetically engineered bacteria and produce green fluorescent protein (GFP). In another report from the same group (Liu et al., 2018), by 3D printing of multiple types of programed cells and chemicals into $3 \mathrm{D}$ architectures and allow communication between different cell types, logic gates, and spatiotemporally responsive patterns were achieved. Each type of cells in the structure perform a simple computational operation, when combined with their spatial distributions in the $3 \mathrm{D}$ architectures, the interactions among different cell types 


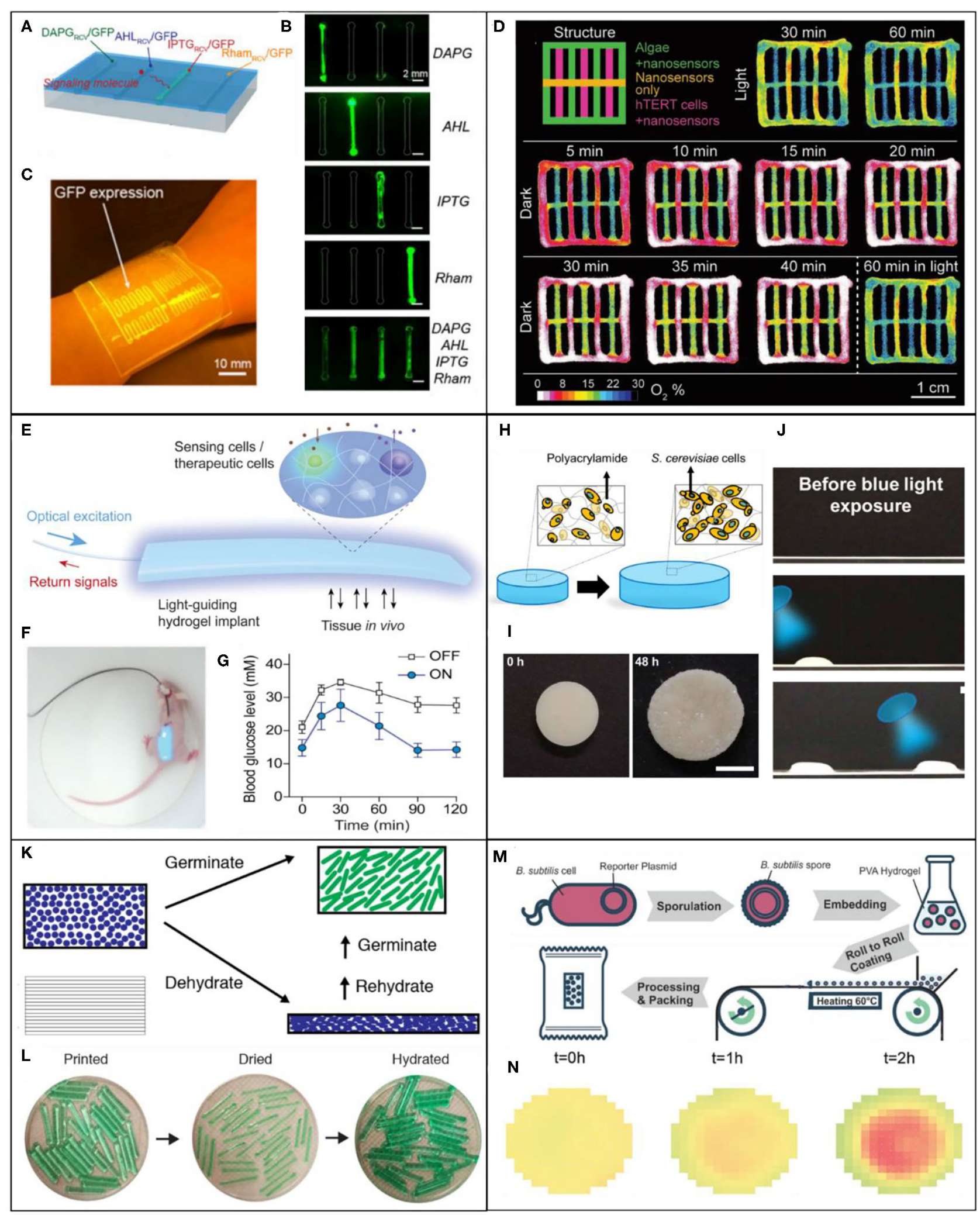

FIGURE 1 | Biological cells incorporated living materials and structures for sensing applications. (A) Schematic illustration of a hydrogel-elastomer hybrid with four isolated chambers to host different bacterial strains. (B) Images of living devices after exposure to individual or multiple inputs, cell chambers hosting bacteria with the 
FIGURE 1 | cognate sensors showed green fluorescence. (C) Photo of a living wearable device based on the hydrogel-elastomer hybrid with encapsulated cells (A-C adapted from Liu et al., 2017). (D) Spatiotemporal dynamics of $\mathrm{O}_{2}$ concentration in a 3D-bioprinted construct consisting of one hydrogel layer of microalgae and sensor nanoparticles (D adapted from Trampe et al., 2018). (E) Schematic of a light-guiding hydrogel encapsulating cells for in vivo sensing and therapy. (F) A blue light coupled hydrogel-implanted mouse in a freely moving state. (G) Blood glucose levels in the chemically induced diabetic mice with and without delivering activation light (E-G adapted from Choi et al., 2013). (H) Schematic of shape change in living composites, the yeast cells proliferate and cause an expansion in the polymer matrix. (I) Macroscopic expansion of a living composite gel with $6 \mathrm{wt} \%$ yeast, scale bar is $7 \mathrm{~mm}$. (J) Patterned photoresponsive living composite with the yeast strain in medium (H-J adapted from Rivera-Tarazona et al., 2020). (K) Schematic of the 3D printed structure composed of hydrogel and B. subtilis spores, which can germinate to perform their engineered function. (L) Reconstitution of the shape of the printed bars that have been dried and then hydrated with water (K,L adapted from González et al., 2020). (M) Schematic showing the bacteria biosensor containing hydrogel production and the application in the roll-to-roll coating production process of the living material. (N) Fluorescence development in the living material after induction with IPTG at three time points (M,N adapted from Schulz-Schönhagen et al., 2019).

and chemicals in different regions induce the emergence of informative patterns and achieve complex logic operations.

The cell metabolic activities in ELMs, as indicated by metabolites levels, including oxygen, carbon dioxide, and glucose, can also be detected when corresponding sensing materials are integrated into the structures. For instance, to convert cell metabolism signals into easily detectable optical signals or color changes (Trampe et al., 2018), hydrogel, luminescent $\mathrm{O}_{2}$-sensing nanoparticles, and living cells were integrated into hybrid bioinks for $3 \mathrm{D}$ printing. (Figure 1D) The fabricated 3D structures enable non-invasive mapping of cell metabolism and spatiotemporal dynamics of their chemical microenvironment. The $\mathrm{O}_{2}$ generated from microalgae or mammalian cells was monitored by the $\mathrm{O}_{2}$-sensitive luminescent indicator molecules using an imaging fluorometer. Optical signals can also be used as an activation mechanism to regulate cell activities and the release of therapeutic compounds (Sankaran and Del Campo, 2019). For instance, cell-integrated hydrogel for in-vivo optical sensing and therapeutic applications was reported (Choi et al., 2013; Figures 1E,F). The cellcontaining hydrogel patches were implanted in awake, freely moving mice, and showed long-term biocompatibility, cellviability, and light-guiding properties. In vivo sensing of nanotoxicity was achieved by using heat-shock-protein 70 , which was activated when exposing to heavy metal ions. More importantly, it was demonstrated that light-controlled optogenetic therapy for type-2 diabetes using the hydrogel patch was possible (Figure 1G); the mice treated with this therapy showed improved glucose homeostasis.

The utilization of fast-growing cells, such as yeast cells, can potentially induce significant shape/size changes to the ELMs with time, which enables a new and straightforward way of biosensing by monitoring the sample shape/size. For instance, the fabrication of living composites materials with the incorporation of yeast cells inside hydrogel was reported (RiveraTarazona et al., 2020; Figures 1H,I). The cellular proliferation leads to a controllable increase in the composite volume of up to $400 \%$. Moreover, spatiotemporally controlled shape change was achieved with pluses of light by encoding an optogenetic switch into the yeast cells (Figure 1J). On the other hand, the coculturing of yeast cells and other types of cells can be used to genetically program biological material assembly. Coculturing of bacteria and yeast was used to fabricate bacterial cellulose-based living materials (Gilbert et al., 2019), the yeast enables DNA-encoded modification of bacterial cellulose. The fabricated living materials can detect and respond to chemical and optical inputs.

In order to use ELMs for more demanding sensing applications, such as long-term environmental monitoring and therapeutic drug monitoring, or process them with fabrication steps that involve harsh conditions, regular living cells embedded in a soft matrix are not stable enough. To overcome this issue, bacterial spores that can survive under unfavorable or even extreme conditions have attracted significant attention recently for the construction of highly durable ELMs. For instance, the fabrication of living materials and structures by $3 \mathrm{D}$ printing with bioinks which contain bacteria spores and hydrogel was reported (González et al., 2020; Figures 1K,L). The incorporation of spores enables its long-term stability and survival in extreme environments, and the spores can germinate on the exterior of the 3D printed structure. By using genetically engineered bacteria, the structure can sense several different kinds of chemicals such as xylose and vanillic acid. In another report (SchulzSchönhagen et al., 2019), a biosensor platform was built based on Bacillus subtilis endospores, which were embedded in a poly(vinyl alcohol) hydrogel matrix with a poly(ethylene terephthalate) packing layer (Figures $\mathbf{1} \mathbf{M}, \mathbf{N}$ ). More importantly, the fabrication process is continuous and scalable, and the biosensing devices can be stored for extended periods of time without losing their functionality. In summary, the integration of ELMs in biosensor devices has provided a new and versatile way to enhance their robustness, biocompatibility, and sensitivity.

\section{ELMS-BASED STRUCTURES FOR ACTUATION}

An actuator is the type of device that can respond to a control signal by converting an energy source into mechanical motion. Soft actuators have been extensively explored in recent years for their applications in soft robotics, biomedicine, and biomimicry (Ware et al., 2015; Zhang et al., 2019b). Conventional soft actuators are usually driven externally by compressed air or pressurized liquids, or based on shape memory polymers or electroactive polymers (Wang et al., 2016; Guin et al., 2018; Rafsanjani et al., 2018). The emergence of ELMs in actuation has the potential to bring new types of soft actuators that are dynamic, fully biocompatible, and self-powered (Dagdeviren et al., 2017; Grattieri and Minteer, 2018; Jeerapan et al., 2019; Moreira et al., 2019; Reid and Mahbub, 2020). 
The incorporation of ELMs in soft actuators can be achieved by using biological cells as the signal-transducing component. A representative design of simple actuator is a gripper which is capable of pick-and-place operation. The fabricated a soft gripper which is capable of converting chemical signals to mechanical movement through the integration of engineered bacteria, flexible light-emitting diode (LED) circuit, and soft pneu-net actuators was reported (Justus et al., 2019; Figures 2A-C). The biosensing soft robot used chemical sensing and feedback to make actionable decisions such as pick-and-place operation.

Certain types of biological cells, including cardiomyocytes and muscle cells, undergo rhythmic contractions, which can be used as the energy source for actuation. For instance, a bioinspired soft-robotic ray was constructed (Park et al., 2016), which is composed of patterned cardiomyocyte tissue on an elastomeric body and a microfabricated gold skeleton (Figure 2D). The combination of optogenetics, tissue engineering, and microfabrication enable phototactic guidance, steering, and turning maneuvers of the artificial ray.

When contractile cells are used in combination with functional optical structures, the mechanical contraction can be converted into optical signal or color changes for easy visualization. For instance, by assembling engineered cardiomyocyte cells on hydrogel films with inverse opal structures (Fu et al., 2018), a hybrid device that can change its photonic bandgap and structural colors during the elongation and contraction process of the cardiomyocytes was demonstrated (Figures 2E,F). Moreover, such hybrid structure can be integrated with microfluidics for direct visualization of microphysiological processes and drug screening (Figures 2G,H).

Recent advances in 3D printing technology, especially direct ink writing (DIW)-based printing, enables the fabrication of sophisticated ELMs-based devices with well-defined 3D structures and integrated components from multiple types of materials. The fabrication of cardiac microphysiological devices via multimaterial 3D printing was reported (Lind et al., 2017; Figures 2I,J), the sensors embedded in the devices allow noninvasive, electronic readouts of tissue contractile stresses. Those devices were also used to study drug responses, as well as the contractile development of human stem cell-derived laminar cardiac tissues over time. To sum up, the biosignal transduction or biomechanical force generated by the biological cells has opened new avenues in the fabrication of functional soft actuators.

\section{CHALLENGES AND FUTURE DIRECTIONS}

A prominent feature of ELMs is that the structure and composition are versatile, dynamic, and are not limited to the properties of synthetic materials alone. The combination of synthetic biology and materials science has the potential to generate ELMs with a wide range of unprecedented chemical compositions, hierarchical structures, and hard-toachieve properties in synthetic materials such as the ability to be self-powered, biosignal-responsive, and self-sustainable. The utilization of ELMs for sensing and actuation has great potential to bring revolutionary changes by providing new sensing and actuation mechanisms based on their tunable responsiveness to biosignals, enhancing the sensitivity, and biocompatibility of the devices by facilitating their integration with biological systems, but there are also issues and challenges that need to be addressed to achieve those objectives.

The genetically engineered cells embedded into a matrix only have limited period of viability without renewable nutrient supply and metabolic waste management. Therefore, for the sensing application of ELMs-based structures, one crucial challenge is how to extend further the life span of the living cells inside the sensors. The utilization of bacterial spores is one possible solution for certain applications (González et al., 2020). But in order to fundamentally overcome this challenge, a more systematic approach to integrate different channels and compartments in the ELMs-based devices for cell sustainability would be necessary. The coculturing and assembly of multiple types of cooperative cells is another promising solution, a good example for this can be found in the metabolic cooperation of multispecies biofilms (Elias and Banin, 2012), where one species utilizes a metabolite produced by a neighboring species, with increased resistance to antibiotics or host immune responses.

The quantitative or precise signal detection and measurements for the ELMs-based sensor still rely mostly on wire connections to external data collection and processing instruments, which limits their applications in areas that require portable or miniaturized devices. The rapid development in micro/nanoelectromechanical systems (MEMS/NEMS) allows the fabrication of miniaturized data collection and processing units. NEMS biosensors correlate chemical or physical binding events with the mechanical motions of devices in nanometer scales, which in turn are converted into detectable electrical or optical signals. They have small form factor, extremely high sensitivity with a detection limit down to the single molecule level (Lim et al., 2015; Ha et al., 2016). The integration of ELMsbased structures with MEMS/NEMS devices has the potential to bring breakthroughs in fully functional and miniaturized ELMs-based sensors.

For the ELMs-based actuators, most of the current work is limited to simple contractile motion or bending. In order to further expand their applications, more sophisticated shape changes or movement need to be realized. This can be potentially achieved by the system-level design of hierarchical 3D structures with the biological cells located at pre-defined locations with different orientations. Patterning and alignment of extracellular matrix with high precision and efficiency are critical to achieving this goal (Kim et al., 2016; Nakayama et al., 2016). Another promising approach is to integrate multiple types of biological cells with stimuli-responsive polymers in an anisotropic and well-controlled manner.

One of the most attractive properties of ELMs is the potential to achieve self-healing, which is intrinsic to all multicellular organisms. But the ELMs-based devices usually also contain synthetic materials and components which are generally not self-healable. In order to achieve self-healing for the whole structure or device, enhance its robustness and lifetime, it is 


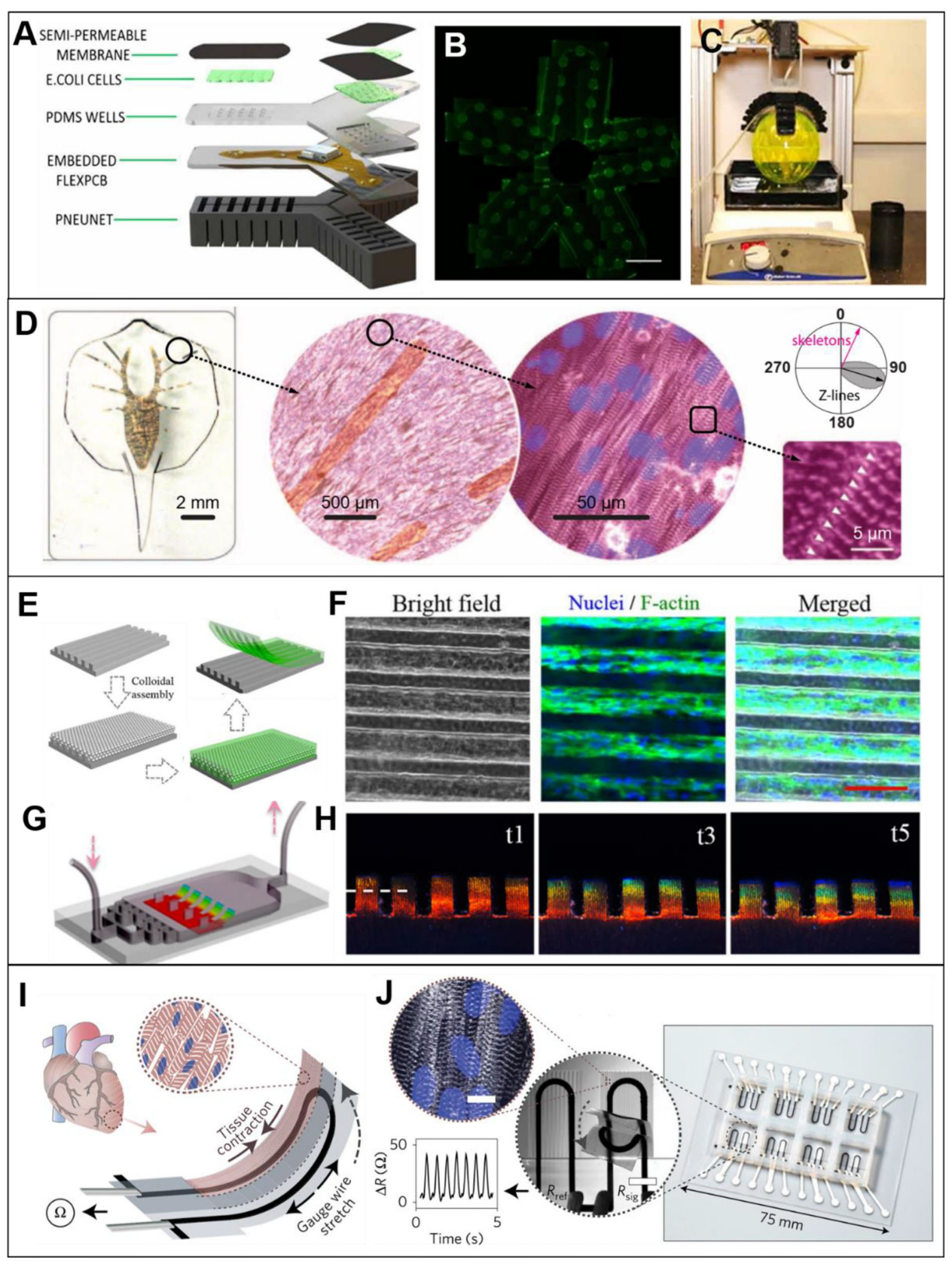

FIGURE 2 | (A) The components of the biosensing soft gripper featured E. coli cells, FlexPCB (flexible printed circuit board), and embedded phototransistors. (B) The gripper contains a genetically engineered $E$. coli layer that show green fluorescence when excited by the embedded LED circuit. (C) The three-finger biogripper 
FIGURE 2 | deploys the object into a bath based on the genetic response of the cells (A-C adapted from Zhang et al., 2019b). (D) System-level design for tissue-engineered ray, which contains a radially oriented muscle cell layer and a composite supporting structure (D adapted form Ware et al., 2015). (E) Schematic diagram of the fabrication process of the microgroove patterned hydrogel films. (F) Confocal microscopy images of the aligned cardiomyocyte tissues on the surface of the inverse opal-structured hydrogel. (G) The structure of the heart-on-a-chip by integrating the biohybrid structural color hydrogel into a microfluidic device. (H) Dynamically optical microscope images of biohybrid structural color hydrogels during one myocardial cycle in a heart-on-a-chip system (E-H adapted from Wang et al., 2016). (I) Schematic of the device made by multimaterial 3D printing, contraction of an anisotropic engineered cardiac tissue induces a resistance change measured by a strain gauge. $(\mathbf{J})$ Image of the final device showing the cardiac tissue on the cantilever surface, insert is the example resistance signal during operation (I,J adapted from Rafsanjani et al., 2018).

important to develop and integrate synthetic self-healing and biocompatible materials with the biological cells (Toohey et al., 2007; Huynh et al., 2017). There has been rapid progress in the development of self-healing polymeric materials in recent years, and the mechanisms include covalent-bond reformation and reshuffling, diffusion and flow, shape-memory effects, supramolecular chemistry, or combinations thereof (Wang and Urban, 2020).

The use of genetically engineered cells is one of the key features for ELMs, which enables the cells to respond to specific signals or secretion of target molecules. Driven by the new methods to design and construct DNA, the genetic engineering of living cells becomes technically easier, at the same time, the functions and behaviors of the engineered cells are getting more complex. Therefore, in order to fabricate ELMs with the precisely targeted functionalities enabled by the genetically engineered cells, it is critical to take advantage of the computational design tools or machine learning to guide the process and predict the final properties. For instance, machine learning algorithms can be used to analyze the huge data sets of genomic sequencing, gene editing, and protein optimization (Shen et al., 2018) to improve the design and function of genetically engineered cells used in ELMs. Moreover, the computational model of the interactions between the component functional materials and cellular/genetic responses is also useful to accelerate the production of functional ELMs-based devices (Smith et al., 2020). Finally, the cost of ELMs-based devices can be quite high, especially when genetic editing or modification is involved. Scaling up of the fabrication process and establishing academia-industry collaboration will be critical for finding viable commercial applications. Finally, the cost of ELMs-based devices can be quite high, especially when genetic editing or modification is involved. Scaling up of the fabrication process and establishing

\section{REFERENCES}

Appiah, C., Arndt, C., Siemsen, K., Heitmann, A., Staubitz, A., and SelhuberUnkel, C. (2019). Living materials herald a new era in soft robotics. Adv. Mater. 31, 1807747. doi: 10.1002/adma.201807747

Arab Hassani, F., Jin, H., Yokota, T., Someya, T., and Thakor, N. V. (2020). Soft sensors for a sensing-actuation system with high bladder voiding efficiency. Sci. Adv. 6:eaba0412. doi: 10.1126/sciadv.aba0412

Bahramzadeh, Y., and Shahinpoor, M. (2014). A review of ionic polymeric soft actuators and sensors. Soft. Rob. 1, 38-52. doi: 10.1089/soro.2013.0006

Bandodkar, A. J., Jeerapan, I., and Wang, J. (2016). Wearable chemical sensors: present challenges and future prospects. ACS Sens. 1, 464-482. doi: 10.1021/acssensors.6b00250 academia-industry collaboration will be critical for finding viable commercial applications.

\section{CONCLUSIONS}

Engineered living materials combine the desired properties of both synthetic soft matter and living biological cells/tissues, and are emerging to the forefront of interdisciplinary research which involves synthetic biology, materials science, device engineering, and robotics. One of the most important and relevant applications of ELMs is for sensing and actuation. In this review, we surveyed and summarized the recent progress of ELMs-based soft sensors and actuators, with a focus on the underlying materials science, interface engineering, and advanced fabrication. The ELMs-based structures and devices have unique advantages over those based only on synthetic materials or biological cells in terms of their sensing mechanism and performance, biocompatibility, versatility, and applicability. We also identified several challenges and issues in this research area and provided possible solutions and future directions for further development of ELMs-based functional structures and devices.

\section{AUTHOR CONTRIBUTIONS}

WX conceived the topic and supervised the manuscript preparation. SL and WX wrote the manuscript. All authors contributed to the article and approved the submitted version.

\section{ACKNOWLEDGMENTS}

We gratefully acknowledge the startup support from the University of Akron.
Bird, L. J., Onderko, E. L., Phillips, D. A., Mickol, R. L., Malanoski, A. P., Yates, M. D., et al. (2019). Engineered living conductive biofilms as functional materials. MRS Commun. 9, 505-517. doi: 10.1557/mrc.2019.27

Cao, Y., Feng, Y., Ryser, M. D., Zhu, K., Herschlag, G., Cao, C., et al. (2017). Programmable assembly of pressure sensors using patternforming bacteria. Nat. Biotechnol. 35, 1087-1093. doi: 10.1038/ nbt. 3978

Caro-Astorga, J., Walker, K. T., and Ellis, T. (2020). Bacterial cellulose spheroids as building blocks for 2D and 3D engineered living materials. bioRxiv. [preprint]. doi: 10.1101/2020.05.11.088138

Chang, H.-J., Voyvodic, P. L., Zúñiga, A., and Bonnet, J. (2017). Microbially derived biosensors for diagnosis, monitoring and epidemiology. Microb. Biotechnol. 10, 1031-1035. doi: 10.1111/1751-7915.12791 
Chen, A. Y., Zhong, C., and Lu, T. K. (2015). Engineering living functional materials. ACS Synth. Biol. 4, 8-11. doi: 10.1021/sb500113b

Chen, D., and Pei, Q. (2017). Electronic muscles and skins: a review of soft sensors and actuators. Chem. Rev. 117, 11239-11268. doi: 10.1021/acs.chemrev.7b00019

Chen, Y., Lu, S., Zhang, S., Li, Y., Qu, Z., Chen, Y., et al. (2017). Skin-like biosensor system via electrochemical channels for noninvasive blood glucose monitoring. Sci. Adv. 3:e1701629. doi: 10.1126/sciadv.1701629

Choi, M., Choi, J. W., Kim, S., Nizamoglu, S., Hahn, S. K., and Yun, S. H. (2013). Light-guiding hydrogels for cell-based sensing and optogenetic synthesis in vivo. Nat. Photonics 7, 987-994. doi: 10.1038/nphoton.2013.278

Cianchetti, M., Laschi, C., Menciassi, A., and Dario, P. (2018). Biomedical applications of soft robotics. Nat. Rev. Mater. 3, 143-153. doi: 10.1038/s41578-018-0022-y

Culver, H. R., Clegg, J. R., and Peppas, N. A. (2018). Analyte-responsive hydrogels: intelligent materials for biosensing and drug delivery. Acc. Chem. Res. 51:2600. doi: $10.1021 /$ acs.accounts.8b00411

Dagdeviren, C., Li, Z., and Wang, Z. L. (2017). Energy Harvesting from the Animal/Human Body for Self-Powered Electronics. Annu. Rev. Biomed. Eng. 19, 85-108. doi: 10.1146/annurev-bioeng-071516-044517

Elias, S., and Banin, E. (2012). Multispecies biofilms: living with friendly neighbors. FEMS Microbiol. Rev. 36, 990-1004. doi: 10.1111/j.1574-6976.2012.00325.x

Fu, F., Shang, L., Chen, Z., Yu, Y., and Zhao, Y. (2018). Bioinspired living structural color hydrogels. Sci. Rob. 3:eaar8580. doi: 10.1126/scirobotics.aar8580

Gilbert, C., and Ellis, T. (2019). Biological engineered living materials: growing unctional materials with genetically programmable properties. ACS Synth. Biol. 8, 1-15. doi: 10.1021/acssynbio.8b00423

Gilbert, C., Tang, T.-C., Ott, W., Dorr, B. A., Shaw, W. M., Sun, G. L., et al. (2019). Living materials with programmable functionalities grown from engineered microbial cocultures. bioRxiv. [preprint]. doi: 10.1101/2019.12.20.882472

González, L. M., Mukhitov, N., and Voigt, C. A. (2020). Resilient living materials built by printing bacterial spores. Nat. Chem. Biol. 16, 126-133. doi: 10.1038/s41589-019-0412-5

Grattieri, M., and Minteer, S. D. (2018). Self-powered biosensors. ACS Sens. 3, 44-53. doi: 10.1021/acssensors.7b00818

Guin, T., Settle, M. J., Kowalski, B. A., Auguste, A. D., Beblo, R. V., Reich, G. W., et al. (2018). Layered liquid crystal elastomer actuators. Nat. Commun. 9:2531. doi: 10.1038/s41467-018-04911-4

Guo, S., Dubuc, E., Rave, Y., Verhagen, M., Twisk, S. A. E., van der Hek, T., et al. (2020). Engineered living materials based on adhesinmediated trapping of programmable cells. ACS Synth. Biol. 9, 475-485. doi: 10.1021 /acssynbio.9b00404

Ha, D., Hong, J., Shin, H., and Kim, T. (2016). Unconventional micro/nanofabrication technologies for hybrid-scale lab-on-a-chip. Lab Chip 16, 4296-4312. doi: 10.1039/C6LC01058J

Heveran, C. M., Williams, S. L., Qiu, J., Artier, J., Hubler, M. H., Cook, S. M., et al. (2020). Biomineralization and successive regeneration of engineered living building materials. Matter 2, 481-494. doi: 10.1016/j.matt.2019.11.016

Heyde, K. C., Scott, F. Y., Paek, S.-H., Zhang, R., and Ruder, W. C. (2017). Using synthetic biology to engineer living cells that interface with programmable materials. J. Vis. Exp. 121:55300. doi: 10.3791/55300

Huang, J., Liu, S., Zhang, C., Wang, X., Pu, J., Ba, F., et al. (2019). Programmable and printable Bacillus subtilis biofilms as engineered living materials. Nat. Chem. Biol. 15, 34-41. doi: 10.1038/s41589-018-0169-2

Huynh, T.-P., Sonar, P., and Haick, H. (2017). Advanced materials for use in soft self-healing devices. Adv. Mater. 29:1604973. doi: 10.1002/adma.201604973

Inda, M. E., and Lu, T. K. (2020). Microbes as biosensors. Annu. Rev. Microbiol. 74, 337-359. doi: 10.1146/annurev-micro-022620-081059

Jeerapan, I., Sempionatto, J. R., and Wang, J. (2019). On-body bioelectronics: wearable biofuel cells for bioenergy harvesting and self-powered biosensing. Adv. Funct. Mater. 30:1906243. doi: 10.1002/adfm.201906243

Justus, K. B., Hellebrekers, T., Lewis, D. D., Wood, A., Ingham, C., Majidi, C., et al. (2019). A biosensing soft robot: autonomous parsing of chemical signals through integrated organic and inorganic interfaces. Sci. Rob. 4:eaax0765. doi: $10.1126 /$ scirobotics.aax 0765

Kim, J., Staunton, J. R., and Tanner, K. (2016). Independent control of topography for 3D patterning of the ECM microenvironment. Adv. Mater. 28, 132-137. doi: 10.1002/adma.201503950
Kim, S., Kim, H., Qiao, T., Cha, C., Lee, S. K., Lee, K., et al. (2019). Fluorescence enhancement from nitro-compound-sensitive bacteria within spherical hydrogel scaffolds. ACS Appl. Mater. Interf. 11, 14354-14361. doi: 10.1021 /acsami.9b02262

Kim, Y., Yuk, H., Zhao, R., Chester, S. A., and Zhao, X. (2018). Printing ferromagnetic domains for untethered fast-transforming soft materials. Nature 558, 274-279. doi: 10.1038/s41586-018-0185-0

Laschi, C., Mazzolai, B., and Cianchetti, M. (2016). Soft robotics: technologies and systems pushing the boundaries of robot abilities. Sci. Rob. 1:eaah3690. doi: 10.1126/scirobotics.aah3690

Le Goff, G. C., Srinivas, R. L., Hill, W. A., and Doyle, P. S. (2015). Hydrogel microparticles for biosensing. Eur. Polym. J. 72, 386-412. doi: 10.1016/j.eurpolymj.2015.02.022

Li, P., Müller, M., Chang, M. W., Frettlöh, M., and Schönherr, H. (2017). Encapsulation of autoinducer sensing reporter bacteria in reinforced alginate-based microbeads. ACS Appl. Mater. Interfaces 9, 22321-22331. doi: $10.1021 /$ acsami.7b07166

Lim, J. W., Ha, D., Lee, J., Lee, S. K., and Kim, T. (2015). Review of micro/nanotechnologies for microbial biosensors. Front. Bioeng. Biotechnol. 3:61. doi: 10.3389/fbioe.2015.00061

Lind, J. U., Busbee, T. A., Valentine, A. D., Pasqualini, F. S., Yuan, H., Yadid, M., et al. (2017). Instrumented cardiac microphysiological devices via multimaterial three-dimensional printing. Nat. Mater. 16, 303-308. doi: $10.1038 / \mathrm{nmat} 4782$

Liu, X., Tang, T.-C., Tham, E., Yuk, H., Lin, S., Lu, T. K., et al. (2017). Stretchable living materials and devices with hydrogel-elastomer hybrids hosting programmed cells. Proc. Natl. Acad. Sci. U.S.A. 114, 2200-2205. doi: 10.1073/pnas.1618307114

Liu, X., Yuk, H., Lin, S., Parada, G. A., Tang, T.-C., Tham, E., et al. (2018). 3D printing of living responsive materials and devices. Adv. Mater. 30:1704281. doi: 10.1002/adma.201704821

Ma, C., Li, J., Zhang, B., Liu, C., Zhang, J., and Liu, Y. (2019). Hydrogel microparticles functionalized with engineered Escherichia coli as living lactam biosensors. Sensors 19:5556. doi: 10.3390/s19245556

Majidi, C. (2018). Soft-matter engineering for soft robotics. Adv. Mater. Technol. 4:1800477. doi: 10.1002/admt.201800477

Miriyev, A., Stack, K., and Lipson, H. (2017). Soft material for soft actuators. Nat. Commun. 8:596. doi: 10.1038/s41467-017-00685-3

Moreira, F. T. C., Frasco, M. F., Barbosa, S. G., Peixoto, L., Madalena Alves, M., and Sales, M. G. F. (2019). Enzymatic self-powered biosensing devices. Bioelectrochem. Interf. Eng. 505-519. doi: 10.1002/9781119611103.ch25

Nakayama, K. H., Surya, V. N., Gole, M., Walker, T. W., Yang, W., Lai, E. S., et al. (2016). Nanoscale patterning of extracellular matrix alters endothelial function under shear stress. Nano. Lett. 16, 410-419. doi: 10.1021/acs.nanolett.5b04028

Nguyen, P. Q., Courchesne, N.-M. D., Duraj-Thatte, A., Praveschotinunt, P., and Joshi, N. S. (2018). Engineered living materials: prospects and challenges for using biological systems to direct the assembly of smart materials. Adv. Mater. 30:e1704847. doi: 10.1002/adma.201704847

Park, S.-J., Gazzola, M., Park, K. S., Park, S., Di Santo, V., Blevins, E. L., et al. (2016). Phototactic guidance of a tissue-engineered soft-robotic ray. Science 353, 158-162. doi: 10.1126/science.aaf4292

Polygerinos, P., Correll, N., Morin, S. A., Mosadegh, B., Onal, C. D., Petersen, K., et al. (2017). Soft robotics: review of fluid-driven intrinsically soft devices; manufacturing, sensing, control, and applications in human-robot interaction. Adv.Eng. Mater. 19:1700016. doi: 10.1002/adem.201700016

$\mathrm{Pu}$, J., Liu, Y., Zhang, J., An, B., Li, Y., Wang, X., et al. (2020). Virus disinfection from environmental water sources using living engineered biofilm materials. Adv. Sci. 7:1903558. doi: 10.1002/advs.201903558

Rafsanjani, A., Zhang, Y., Liu, B., Rubinstein, S. M., and Bertoldi, K. (2018). Kirigami skins make a simple soft actuator crawl. Sci. Rob. 3:eaar7555. doi: 10.1126/scirobotics.aar7555

Reid, R. C., and Mahbub, I. (2020). Wearable self-powered biosensors. Curr. Opin. Electrochem. 19, 55-62. doi: 10.1016/j.coelec.2019.10.002

Rich, S. I., Wood, R. J., and Majidi, C. (2018). Untethered soft robotics. Nat. Electron. 1, 102-112. doi: 10.1038/s41928-018-0024-1

Ricotti, L., Trimmer, B., Feinberg, A. W., Raman, R., Parker, K. K., Bashir, R., et al. (2017). Biohybrid actuators for robotics: a review of devices actuated by living cells. Sci. Rob. 2:eaaq0495. doi: 10.1126/scirobotics.aaq0495 
Rivera-Tarazona, L. K., Bhat, V. D., Kim, H., Campbell, Z. T., and Ware, T. H. (2020). Shape-morphing living composites. Sci. Adv. 6:eaax8582. doi: $10.1126 /$ sciadv.aax 8582

Roggo, C., and van der Meer, J. R. (2017). Miniaturized and integrated whole cell living bacterial sensors in field applicable autonomous devices. Curr. Opin. Biotechnol. 45, 24-33. doi: 10.1016/j.copbio.2016.11.023

Ruan, X., Wang, Y., Cheng, N., Niu, X., Chang, Y., Li, L., et al. (2020). Emerging applications of additive manufacturing in biosensors and bioanalytical devices. Adv. Mater. Technol. 5:2000171. doi: 10.1002/admt.202000171

Sankaran, S., and Del Campo, A. (2019). Optoregulated protein release from an engineered living material. Adv. Biosyst. 3:e1800312. doi: 10.1002/adbi.201800312

Schulz-Schönhagen, K., Lobsiger, N., and Stark, W. J. (2019). Continuous production of a shelf-stable living material as a biosensor platform. Adv. Mater. Technol. 4:1900266. doi: 10.1002/admt.201900266

Shen, M. W., Arbab, M., Hsu, J. Y., Worstell, D., Culbertson, S. J., Krabbe, O., et al. (2018). Predictable and precise template-free CRISPR editing of pathogenic variants. Nature 563, 646-651. doi: 10.1038/s41586-018-0686-x

Smith, R. S. H., Bader, C., Sharma, S., Kolb, D., Tang, T., Hosny, A., et al. (2020). Hybrid living Materials: digital design and fabrication of 3D multimaterial structures with programmable biohybrid surfaces. Adv. Funct. Mater. 30:1907401. doi: 10.1002/adfm.201907401

Su, L., Jia, W., Hou, C., and Lei, Y. (2011). Microbial biosensors: a review. Biosens. Bioelectron. 26, 1788-1799. doi: 10.1016/j.bios.2010.09.005

Toohey, K. S., Sottos, N. R., Lewis, J. A., Moore, J. S., and White, S. R. (2007). Self-healing materials with microvascular networks. Nat. Mater. 6, 581-585. doi: 10.1038/nmat1934

Trampe, E., Koren, K., Akkineni, A. R., Senwitz, C., Krujatz, F., Lode, A., et al. (2018). Functionalized bioink with optical sensor nanoparticles for O2 imaging in 3D-bioprinted constructs. Adv. Funct. Mater. 28:1804411. doi: 10.1002/adfm.201804411

Trantidou, T., Dekker, L., Polizzi, K., Ces, O., and Elani, Y. (2018). Functionalizing cell-mimetic giant vesicles with encapsulated bacterial biosensors. Interface Focus 8:20180024. doi: 10.1098/rsfs.2018.0024
Truby, R. L., Wehner, M., Grosskopf, A. K., Vogt, D. M., Uzel, S. G. M., Wood, R. J., et al. (2018). Soft somatosensitive actuators via embedded 3D printing. Adv. Mater. 30:e1706383. doi: 10.1002/adma.201706383

Velasco, D., Tumarkin, E., and Kumacheva, E. (2012). Microfluidic encapsulation of cells in polymer microgels. Small 8, 1633-1642. doi: 10.1002/smll.201102464

Wang, M., Lin, B.-P., and Yang, H. (2016). A plant tendril mimic soft actuator with phototunable bending and chiral twisting motion modes. Nat. Commun. 7:13981. doi: 10.1038/ncomms13981

Wang, S., and Urban, M. W. (2020). Self-healing polymers. Nat. Rev. Mater. 5, 562-583. doi: 10.1038/s41578-020-0202-4

Ware, T. H., McConney, M. E., Wie, J. J., Tondiglia, V. P., and White, T. J. (2015). Actuating materials. Voxelated liquid crystal elastomers. Science 347, 982-984. doi: 10.1126/science.1261019

Xu, W., Paidi, S. K., Qin, Z., Huang, Q., Yu, C.-H., Pagaduan, J. V., et al. (2019). Self-folding hybrid graphene skin for 3D biosensing. Nano Lett. 19, 1409-1417. doi: 10.1021/acs.nanolett.8b03461

Zhang, Y., Ren, T., He, J., Tian, H., and Jin, B. (2019a). Acute heavy metal toxicity test based on bacteria-hydrogel. Colloids Surf. A. 563, 318-323. doi: 10.1016/j.colsurfa.2018.12.016

Zhang, Y., Zhang, N., Hingorani, H., Ding, N., Wang, D., Yuan, C., et al. (2019b). Fast-response, stiffness-tunable soft actuator by hybrid multimaterial 3D printing. Adv. Funct. Mater. 29:1806698. doi: 10.1002/adfm.20180 6698

Conflict of Interest: The authors declare that the research was conducted in the absence of any commercial or financial relationships that could be construed as a potential conflict of interest.

Copyright (C) $2020 \mathrm{Liu}$ and Xu. This is an open-access article distributed under the terms of the Creative Commons Attribution License (CC BY). The use, distribution or reproduction in other forums is permitted, provided the original author(s) and the copyright owner(s) are credited and that the original publication in this journal is cited, in accordance with accepted academic practice. No use, distribution or reproduction is permitted which does not comply with these terms. 